\title{
Studying the Interaction of 10-keV Electrons with a Dielectric Surface
}

\author{
K. A. Vokhmyanina ${ }^{a}$, P. N. Zhukova ${ }^{a}$, A. S. Kubankin ${ }^{a}$, Le Thu Hoai ${ }^{a}$, R. M. Nazhmudinov ${ }^{a}$, \\ A. A. Pleskanev ${ }^{a}$, A. N. Oleinik ${ }^{a}$, N. N. Nasonov ${ }^{a}$, and G. P. Pokhil ${ }^{b}$ \\ ${ }^{a}$ Laboratory of Radiation Physics, Belgorod State University, Belgorod, Russia \\ ${ }^{b}$ Skobeltsyn Research Institute of Nuclear Physics, Moscow State University, Moscow, Russia \\ Received July 17, 2013
}

\begin{abstract}
The grazing incidence interaction of a 10-keV electron beam with a planar surface of plexiglass is studied experimentally. Moreover, the electron passage through flat channels formed by such surfaces is investigated. The experiments reveal the presence of a guiding effect of the electron passage as in the case of a glass surface. However, there are some features, such as the existence of an initial elevation angle for the case of negative inclination angles of the plate. The formation of self-consistent charge on the surface of the plexiglass and its drain when the current is turned off occur more slowly than on the glass surface. This fact points to the difference in the surface conductivity of insulators.
\end{abstract}

DOI: $10.1134 / \mathrm{S} 1027451014020438$

\section{INTRODUCTION}

Studies into the effect of motion control of an electron beam by means of dielectric channels were started relatively recently [1-4]. It was supposed that a part of an incident electron beam when passing through a dielectric channel forms a self-organizing system of charges providing contactless passage of the rest of the beam. Moreover, this mechanism is also implemented upon rotation of the dielectric channel relative to a beam of electrons with energies up to $1 \mathrm{keV}$.

To simplify analysis of this mechanism, studying the interaction of electrons with glass surfaces at grazing incidence was carried out [5]. Experiments with glass plates revealed some features of the process as compared to analogous experiments with ion beams: a dependence of the reflection on the length of the plate and the presence of a beam trace halo on the screen.

To find out how the dielectric material affects the nature of the charge distribution and the rate of its formation, we have continued studying planar surfaces made of plexiglass (polymethylmethacrylate).

\section{EXPERIMENT}

The experimental scheme is shown in Fig. 1. The main units of the setup were made of nonmagnetic materials to eliminate the possibility of the effect of a magnetic field on the electron beam. The samples were fixed to a goniometer which allowed them to be tilted at angles up to 10 degrees relative to the beam axis and to be displaced in the horizontal plane, making it possible to simultaneously work with several samples and adjust the straight beam. A glass screen covered by a scintillator with a layout of $1.5 \mathrm{~cm} \times 1.5 \mathrm{~cm}$ was mounted at a distance of 24.5 or $32 \mathrm{~cm}$ from the axis of the beam depending on the experimental scheme. The motion of the beam trace on the screen was recorded by means of a webcam located outside the vacuum chamber. The sample was rotated successively by means of the goniometer and photos were taken at each fixed angle.

\section{Dielectric Surface}

An electron beam with an energy of $E=10 \mathrm{keV}$, a mask current of $100 \mathrm{nA}$, a beam diameter cross section of about $2 \mathrm{~mm}$, and a divergence of $<0.2^{\circ}$ was incident onto a planar dielectric surface at a glancing angle. The electron trace is created on the screen after interaction and is recorded using the webcam. Figure 2 shows a series of images demonstrating displacement of the beam trace on the screen when the plexiglass plate of $50 \mathrm{~mm}$ length was tilted at angles ranging from $-1^{\circ}$ to $2.8^{\circ}$ relative to the beam axis. The front part of the plate was covered with a grounded aluminum foil. The bright line of the photo shows the trace level of the straight beam. The distance to the screen is $24.5 \mathrm{~cm}$.

On the whole, the character of spot motion on the screen is similar to that when turning the flat surface of the glass. However, the formation of self-consistent charge on the surface of the plexiglass (forming the stable trace on the screen) and its drain when the current is switched off occur slower (up to $10 \mathrm{~min}$ ) than on the glass surface, indicating the difference in the surface conductivity of these dielectrics. 


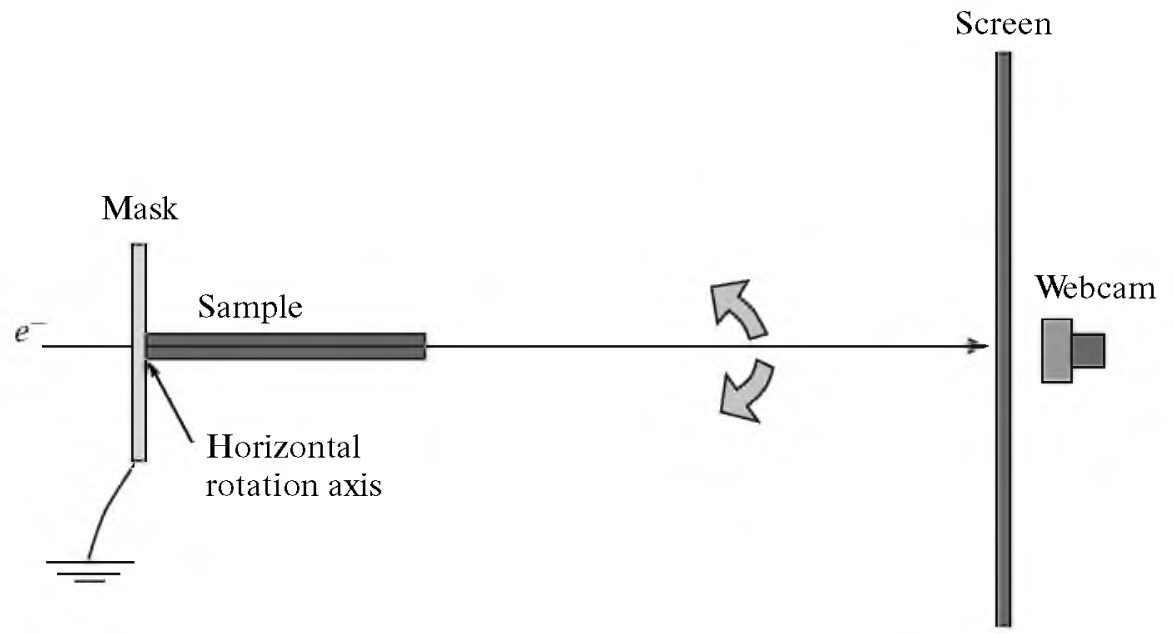

Fig. 1. General scheme of the experiments.

In some experiments, as in the case of the glass surface, the trace of the beam on the screen bifurcates onto two parts (Fig. 3a). The position of the first one coincides with the trace of the straight beam. The second part represents a strongly scattered spot ("cloud") or an arch which ascends in accordance with the plate turning relative to the beam axis (Fig. 3b). The transverse size of the incident beam is about $2 \mathrm{~mm}$ and the length of the plate studied is $50 \mathrm{~mm}$.

The shift of the trace of the "cloud" on the screen was measured according to the displacement of the lower border of the spot relative to the zero position of the plate (parallel to the beam). The shape of the "cloud" is likely dependent on the processing quality of the plate and the beam-current magnitude. Figure 3a shows the photo obtained in the case of a matte plexiglass surface interacting with a current of $\sim 100 \mathrm{nA}$.

A large number of the experiments with dielectric surfaces showed ascension of the beam trace even at negative inclination angles of the plates relative to the beam axis. To find the cause of beam-trace ascension, the output part of the plate was grounded as shown in Fig. 4. The rear end of the plate and $1 \mathrm{~mm}$ of the reflecting surface were covered by the grounded foil. In this case, the beam ascension on the screen disappeared or the trace was shifted below the trace of the straight beam.

Figure 5 shows the graphs for plates with grounded and nongrounded output surfaces. The length of the plates and the current are equal to $35 \mathrm{~mm}$ and $40 \mathrm{nA}$.

The difference in the positions of the traces for identical plates can be explained by the different magnitude of the electron current incident on the surface, since the experiment was conducted so that a part of the beam was incident on the surface at a glancing angle, and correspondingly a part of the beam bombarded the grounded front end.

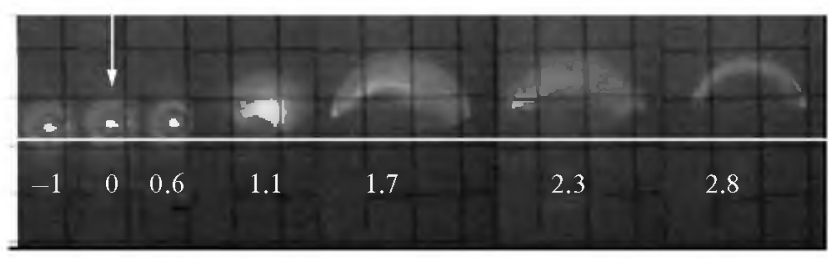

Fig. 2. Displacement of the beam trace on the screen by tilting a plate of $5 \mathrm{~cm}$ length by angles from $-1^{\circ}$ to $2.8^{\circ}$ relative to the beam axis.

(a)
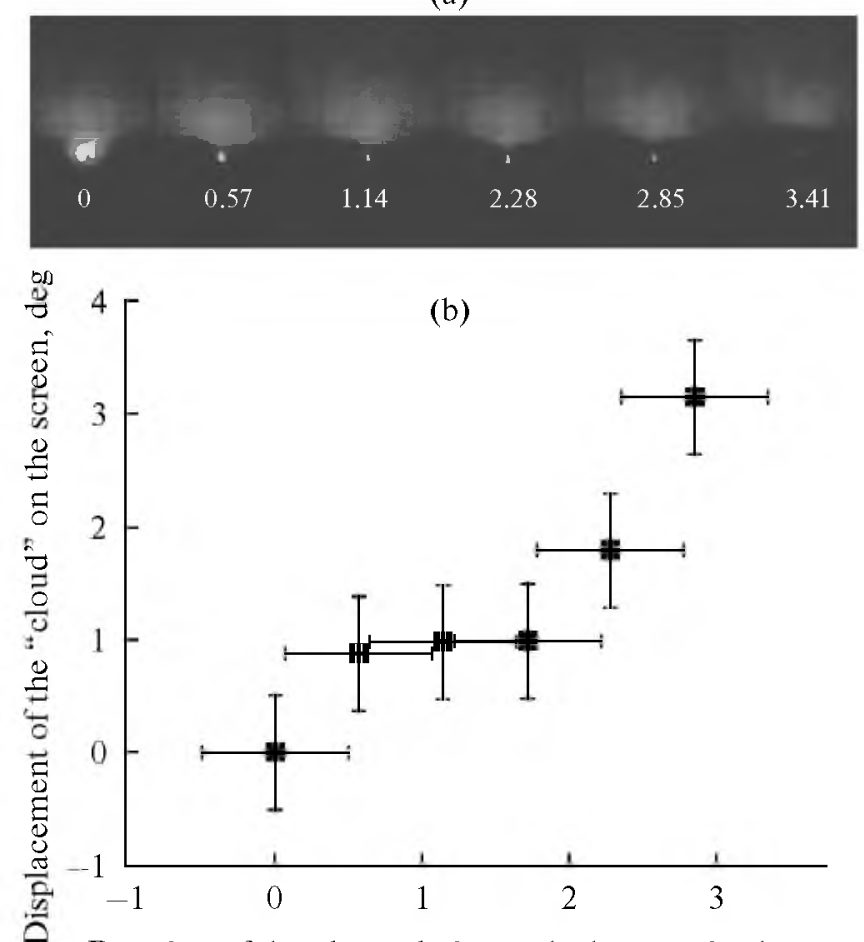

Rotation of the plate relative to the beam axis, deg

Fig. 3. Separation of the trace of the beam on the screen into two parts upon inclination of the plate of plexiglass $5 \mathrm{~cm}$ in length (a). Dependence of the spot motion on the tilt angle of the plate (b). 
(a)

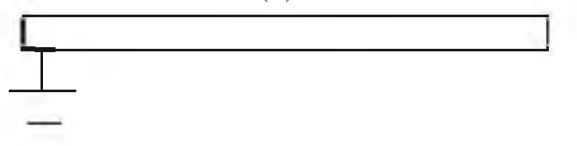

(b)

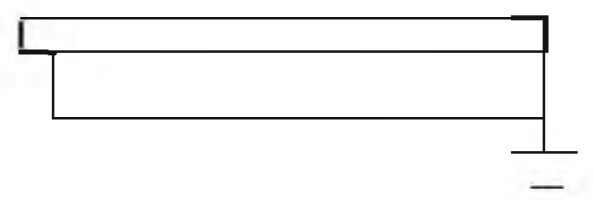

Fig. 4. Scheme of the plate grounding (side view): (a) only the front end is grounded; (b) both ends are grounded and the part of the surface on the output.
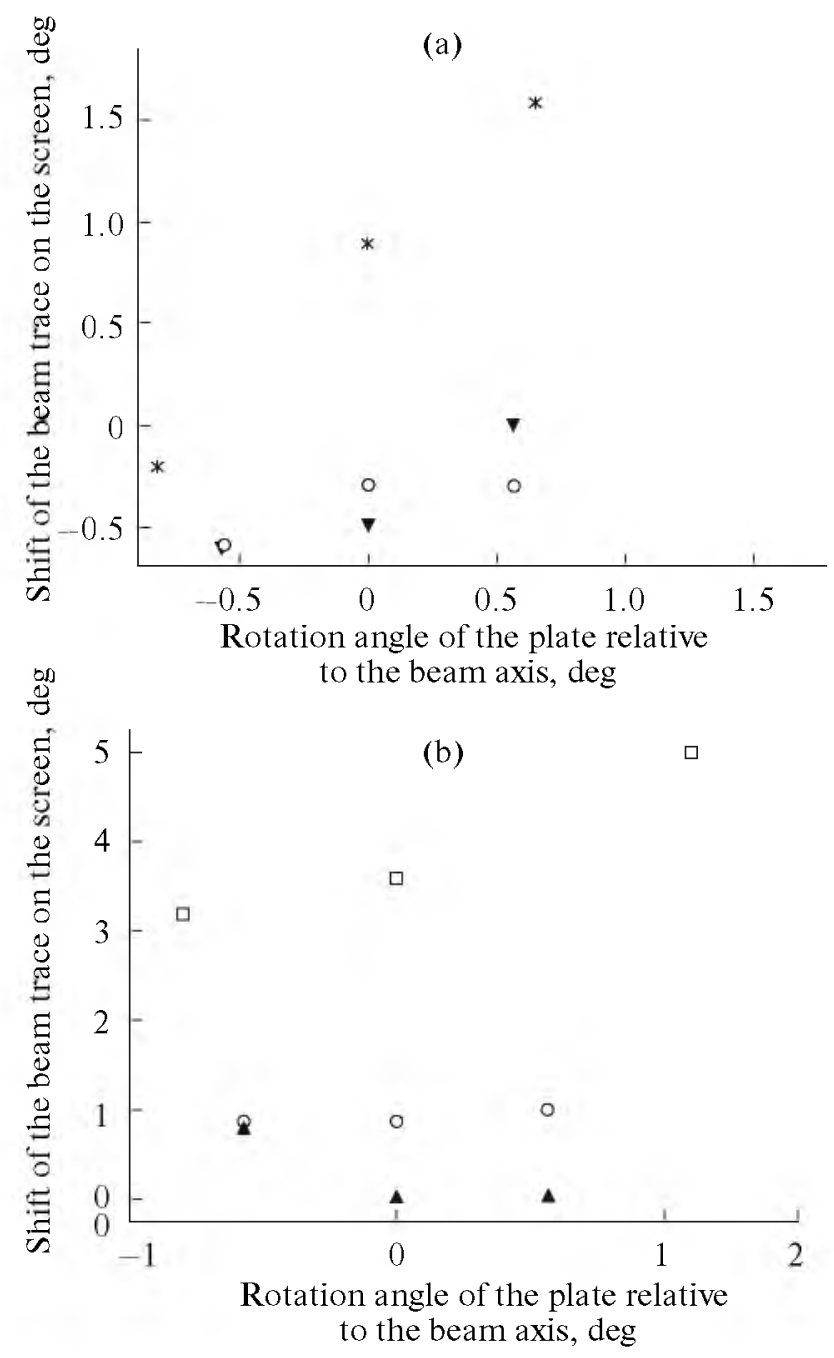

Fig. 5. Motion track of the beam depending on the tilt angle in the case of grounding: both ends of the plate and part of the reflecting surface at the output (a); only the front end (b). The length of the plates is equal to $35 \mathrm{~mm}$. In each series, shown are the curves obtained for different brands of plexiglass.

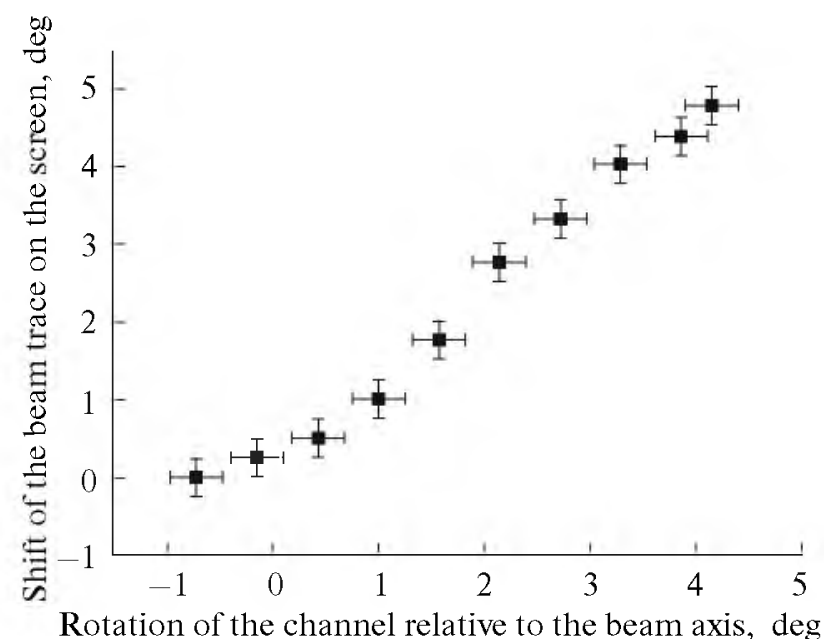

Fig. 6. Dependence of the beam trace on the screen from the tilt angle of the dielectric planar channel of $35 \mathrm{~mm}$ length.

\section{Flat Plexiglass Channel}

Figure 6 shows the dependence of the position of the beam trace on the screen on the tilt angle of the planar dielectric channel made of plexiglass. The channel length is $35 \mathrm{~mm}$, the distance between the plates is $\sim 1 \mathrm{~mm}$, and the current at the input part of the sample equals $200 \mathrm{nA}$. As we can see from the figure, similar to cylindrical plastic channels [5], the electron beam is effectively controlled by the planar channel in the limits of 4 without appreciable loss in intensity.

\section{DISCUSSION OF THE RESULTS}

The results of our experiments demonstrated that, as in the case of electron interaction with a glass surface, self-consistent charge distribution is formed on the surface of organic glass allowing electrons to move together with the plates and planar channels for the case of small rotation angles relative to the beam axis.

An atypical dependence of the spots on the screen at angles close to zero relative to the axis of the beam can be attributed to the formation of oppositely charged structures on the surface of the glass due to the large output of secondary electrons at grazing angles of incidence. Unlike creating image charges [6], this mechanism does not need high currents and may completely explain the behavior of the electron beam at negative tilt angles of the plates. This problem is the topic of our next investigation and can be solved by means of computer simulation based on the string model described in [7]. 


\section{CONCLUSIONS}

As a result of experiments on the planar surface of plexiglass, we have observed the effect of motion control of an electron beam, as in the case of a glass surface. However, some features were revealed in the case of plexiglass, namely, the rate of the formation of the stable charge state on the dielectric surface and the presence of initial trace ascension at negative tilt angles of the plate. In some cases, we observed lowering of the beam trace on the screen below the track of the straight beam at small angles of inclination of the plate relative to the beam.

We have also demonstrated motion control of an electron beam in the case of planar plexiglass channels.

\section{REFERENCES}

1. S. Das, B. S. Dassanayake, M. Winkworth et al,, Phys. Rev. A 76, 042716 (2007).
2. K. Schiessl, C. Lemell, K. Tökési, and J. Burgdörfer, J. Phys. Conf. Ser. 194, 012069 (2009).

3. V. P. Petukhov, J. Surf. Invest.: X-Ray, Synchrotron Neutron Tech. 4, 189 (2010).

4. W. Wang, J. Chen, D. Y. Yu, B. Yang, et al., Phys. Scr. 144, 014023 (2011).

5. K. A. Vokhmyanina, P. N. Zhukova, E. F. Irribarra, et al., J. Surf. Invest.: X-Ray, Synchrotron Neutron Tech. 7, 271 (2013).

6. K. A. Vokhmyanina, P. N. Zhukova, Le Tkhi Khoai, et al., J. Surf. Invest.: X-Ray, Synchrotron Neutron Tech. 6, 266 (2012).

7. G. P. Pokhil and V. V. Cherdyntsev, J. Surf. Invest.: X-Ray, Synchrotron Neutron Tech. 7, 356 (2013).

Translated by G. Dedkov 